\title{
Label-free Classification of Bacterial Extracellular Vesicles by Combining Nanoplasmonic Sensors with Machine Learning
}

This paper was downloaded from TechRxiv (https://www.techrxiv.org).

\section{LICENSE}

CC BY 4.0

SUBMISSION DATE / POSTED DATE

24-08-2021 / 26-08-2021

\section{CITATION}

Kazemzadeh, Mohammadrahim; Hisey, Colin; Dauros Singorenko, Priscila; Swift, Simon; Zargar, Kamran; Xu, Peter; et al. (2021): Label-free Classification of Bacterial Extracellular Vesicles by Combining Nanoplasmonic Sensors with Machine Learning. TechRxiv. Preprint. https://doi.org/10.36227/techrxiv.16432926.v1

$\mathrm{DOI}$ 


\title{
Label-free Classification of Bacterial Extracellular Vesicles by Combining Nanoplasmonic Sensors with Machine Learning
}

\author{
Mohammadrahim Kazemzadeh, Colin L. Hisey*, Priscila Dauros-Singorenko, Simon Swift, Kamran \\ Zargar-Shoshtari, Weiliang Xu, Senior-Member, IEEE, Neil G.R. Broderick
}

\begin{abstract}
Bacterial extracellular vesicles (EVs) are nanoscale lipidenclosed packages that are released by bacteria cells and shuttle various biomolecules between bacteria or host cells. They are implicated in playing several important roles, from infectious disease progression to maintaining proper gut health, however the tools available to characterise and classify them are limited and impractical for many applications. Surface-enhanced Raman Spectroscopy (SERS) provides a promising means of rapidly fingerprinting bacterial EVs in a label-free manner by taking advantage of plasmonic resonances that occur on nanopatterned surfaces, effectively amplifying the inelastic scattering of incident light. In this study, we demonstrate that by applying machine learning algorithms to bacterial EV SERS spectra, EVs from cultures of the same bacterial species (Escherichia coli) can be classified by strain, culture conditions, and purification method. While these EVs are highly purified and homogeneous compared to complex samples, the ability to classify them from a single species demonstrates the incredible power of SERS when combined with machine learning, and the importance of considering these parameters in future applications. We anticipate that these findings will play a crucial role in developing the laboratory and clinical utility of bacterial EVs, such as the label-free, noninvasive, and rapid diagnosis of infections without the need to culture samples from blood, urine, or other fluids.

Index Terms_-Plasmonic, SERS, biosensor, extracellular vesicles, Raman spectroscopy, nonlinear optics, Escherichia coli, outer membrane vesicles, exosomes.

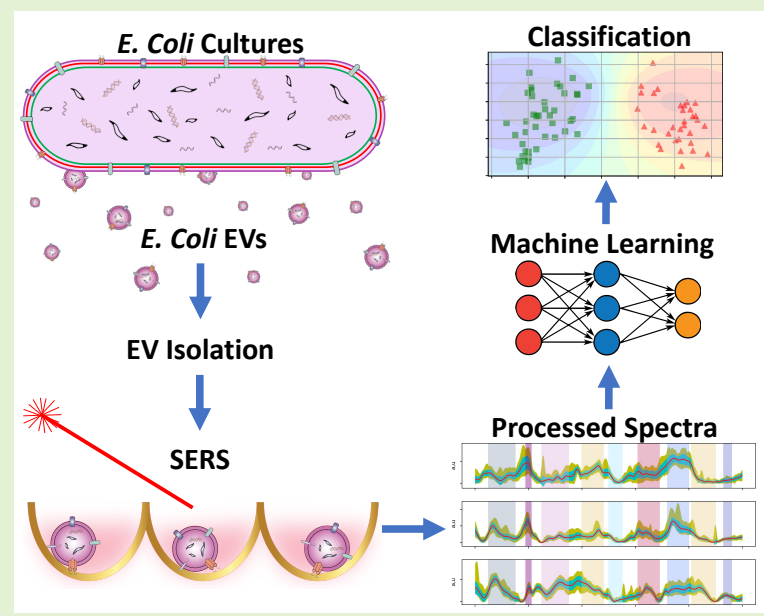

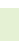

\section{INTRODUCTION}

This article was submitted on August 24, 2021. Funding support information can be found in the acknowledgements. The first two authors contributed equally to this work. Asterisk indicates corresponding author.

M. K. is with the Department of Mechanical Engineering and the Dodd-Walls Centre for Photonic and Quantum Technologies, University of Auckland, New Zealand (e-mail: mkaz499@aucklanduni.ac.nz).

${ }^{*} \mathrm{C}$. $\mathrm{H}$. is with the Hub for Extracellular Vesicle Investigations and the Department of Obstetrics and Gynaecology, University of Auckland, New Zealand. (e-mail: colin.hisey@auckland.ac.nz).

P. D. is with the Department of Molecular Medicine and Pathology and School of Biological Sciences at the University of Auckland, New Zealand (e-mail: p.dauros@auckland.ac.nz).

S. S. is with the Department of Molecular Medicine and Pathology at the University of Auckland, New Zealand (e-mail: s.swift@auckland.ac.nz).

K. Z. is with the Department of Surgery at the University of Auckland, New Zealand (e-mail: kamran.zargar@auckland.ac.nz).

W. X. is with the Department of Mechanical Engineering, University of Auckland, New Zealand (e-mail: p.xu@auckland.ac.nz).

N. B. is with the Department of Physics and the Dodd-Walls Centre for Photonic and Quantum Technologies, University of Auckland, New Zealand (e-mail: n.broderick@auckland.ac.nz). 
troscopy, techniques such as Surface-enhanced Raman Spectroscopy (SERS) [13] and laser tweezers Raman spectroscopy (LTRS) [14] have been applied to characterising bacteria cells. SERS benefits from strong plasmonic interactions of incident laser light with the electrons of noble metals on a substrate's surface [15], [16], creating strong localised electric fields at specific hot spots by confining the light in the nanoscale geometric surface features. In doing so, any nonlinear lightmaterial interactions, including Stokes and anti-Stokes inelastic scattering, are substantially amplified. This technique is capable of identifying biomolecules at extremely low concentrations and is even capable of single molecule detection. Plasmonic nanoparticles conjugated with antibodies have also been used in immunoassays for the selective detection of E. coli from complex fluid samples [17]. In addition, SERS has been used for the label-free detection of $E$. coli strain classification by the direct acquisition of the Raman signal of cells and subsequent multivariate analysis techniques [18].

Recently, there has been growing interest in using SERS for both labeled [19]-[24] and label-free [25]-[30] characterisation of mammalian cell-derived extracellular vesicles (EVs). Bacteria, as their eukaryotic counterparts, release EVs, which are lipid-enclosed nanoscale packages that are derived from the membranes of parental cells and can harbour a diverse molecular cargo such as proteins, DNA, RNA, glycolipids, organic small molecules, etc [31]. Therefore, analysing bacterial EVs using SERS could provide valuable information related to a range of biomolecules and help identify the condition of the parental bacteria cells. Although EV isolation techniques have become more rapid, less laborious, and require less expensive or specialised equipment, leading to the development of novel downstream characterisation tools including SERS, bacterial EV research itself still faces a multitude of experimental-dependent factors. Most bacterial $\mathrm{EV}$ research has been performed in vitro, where hundreds of different culture conditions (e.g. media, growth mode or stage, temperature, oxygen, +/- stimulus) along with different $\mathrm{EV}$ isolation methods can produce significantly different EV compositions, mainly quantified based on protein [32]-[34] and RNA [35]-[37] content.

Recently, SERS has proven to be a promising method for early detection and classification of various diseases such as cancer [38], [39]. While all bacteria release EVs into their surrounding space, translating into numerous biological roles in their hose or their own life cycles [40]-[51], circulating bacterial EVs may also hold potential to be used as a substrate for infection detection and identification. Importantly, the rapid growth pace of bacteria allows cells to release EVs in quick response to changes in environmental cues, resulting in $\mathrm{EV}$ molecular compositions that may represent specific temporal or environmental conditions [33]. The bacterial EV field has focused mainly on conventional proteomics-based methods of bacterial EV characterisation, as bacterial EV proteins have shown to be diverse, abundant, highly responsive to environmental conditions, and informative about biogenesis mechanisms or potential virulence roles [52]. More recently, RNA-seq has also provided great utility in characterising bacterial EV RNA content, however RNA's variable presence or low abundance in EVs, combined with time-consuming methods and advanced equipment or skills needed restrict efficient progress. Clearly, there is a need for a platform enabling analysis and profiling across all molecular cargo present in bacterial EVs, and SERS could provide a powerful, comprehensive, and sensitive tool to characterise them but potentially to also detect or even classify their parental bacteria cells' identity or condition.

To date, only one recent study demonstrated the use of conventional Raman spectroscopy for characterising bacterial EVs, where changes in the chemical profiles of Pseudomonas chlororaphis $\mathrm{O} 6$ (PcO6) cells and resultant EVs were identified according to different abiotic stressors. In addition, they showed that the bacterial EVs had higher relative concentrations of proteins, lipids, and nucleic acids than PcO6 cells [53]. However, to the best of our knowledge, no studies to date have examined whether bacterial EVs could be successfully fingerprinted or classified using SERS.

In this study, we demonstrate for the first time, that by combining label-free SERS with machine learning, E. coli EVs can be classified based on differences in strain, culture condition, and purification method. Probiotic (Nissle), uropathogenic (UPEC), and laboratory model (K12) E. coli strains were classified in both pairwise and collective approaches. In addition, manifold machine learning proved to be a viable method of dimension reduction for EV SERS spectra visualisation. We believe that these results will encourage and support other researchers towards utilising SERS for bacterial EV characterisation in a multitude of laboratory and clinical applications.

\section{Materials and Methods}

\section{A. Bacteria Culture and EV Isolation}

Three Escherichia coli strains were used for this study: Uropathogenic E. coli (UPEC) strain 536 (O6:K15:H31) [54], probiotic Nissle 1917 [55] and laboratory model strain MG1655 (K-12, ATCC® 47076) [56]. Culture and EV isolation methods have been previously published in detail [33]. Briefly, bacterial cells were grown in either of two iron conditions: iron restricted, in plain RPMI 1640 medium (R) (Thermo Fisher Scientific) or iron sufficient, in RPMI medium supplemented with $10 \mu \mathrm{M}$ iron(III) chloride (RF) to better reflect physiological conditions. At the desired incubation time, bacterial cells were removed from the culture by centrifugation and filtration. Cell-free EV-containing supernatants were concentrated to smaller volumes with $100 \mathrm{kDa}$ Vivaflow 200 cassettes (Sartorius AG) and EVs were pelleted by ultracentrifugation at $75,000 \times g$ for $2.5 \mathrm{~h}$ at $4{ }^{\circ} \mathrm{C}$ (Beckman Avanti J-30I), then resuspended in PBS. EVs were then further purified with either of two well-established purification methods: Density Gradient Centrifugation (DG) using an iodixanol (Optiprep, SigmaAldrich) gradient or Size Exclusion Chromatography (SEC) using a qEV Original column (70 nm, Izon Science) [33]. For the remainder of this study, EV samples are referred to using the notation "Strain-Culture Medium-Purification Method". For example, EVs from UPEC cells grown with iron supplementation and purified with size exclusion chromatography are referred to as "UPEC-RF-SEC". 


\section{B. EV Characterisation}

EV-rich fractions from both SEC and DG purification methods were determined by protein (Pierce ${ }^{\mathrm{TM}}$ BCA Protein Assay, ThermoFisher) and particle quantification using nanoparticle tracking analysis (NTA) using an NS300 Nanosight (Malvern Panalytical), then pooled for analysis. Once EV-rich fractions were pooled, they were diluted at a 1:250 ratio in PBS and three 30 second videos were taken under low flow conditions (Screen gain:2, Camera level:14) and characterised using the Nanosight 3.4 software (Screen gain:10, Detection threshold:6) to calculate mean and mode particle diameters, concentration, and size distributions. Prior to TEM or SERS, EV samples were transferred from PBS buffer to ultrapure water by loading $200 \mu \mathrm{l}$ of the purified EVs into a Vivaspin 500 (Sartorius AG) centrifugal concentrator with a $100 \mathrm{kDa}$ cutoff and centrifuging at $10,000 \times g$ until most of the PBS had flowed through the filter (roughly 10 minutes). Ultrapure water (ThermoFisher), $450 \mu \mathrm{l}$, was then added to the filter and the centrifugation process was repeated twice more before finally suspending the EVs in $100 \mu \mathrm{l}$ ultrapure water. Negative staining TEM of purified EVs was conducted by adsorption onto Formvar-coated copper grids (Electron Microscopy Sciences) for 10 minutes. Excess liquid was removed with filter paper (Whatman) and the copper grid was then transferred to $20 \mu \mathrm{L}$ of $2 \%$ filtered uranyl acetate for 2 minutes. Excess liquid was again removed with filter paper and the grid was allowed to dry under a lamp for 10 minutes. Grids were visualised on Tecnai G2 Spirit TWIN (FEI, Hillsboro, OR, USA) transmission electron microscope (TEM) at $120 \mathrm{kV}$ accelerating voltage. Images were captured using a Morada digital camera (SIS GmbH, Munster, Germany).

\section{SERS Preparation, Characterisation, and Measurements}

A gold plasmonic microresonator was fabricated as previously reported [57]-[60]. Briefly, $1 \mu \mathrm{m}$ polystyrene beads (Sigma) were carefully spread onto glass slides and baked, then a PDMS mold was taken of the resulting bead monolayer. Following demolding, the PDMS was sonicated in acetone and isopropyl alcohol to remove residual beads and baked overnight. Gold was deposited by sputter coating (Q150R S, Quorum) with the rate of $0.5 \mathrm{~nm}$ per minute until a $12.5 \mathrm{~nm}$ thickness was achieved, then the deposition rate was increased to $6 \mathrm{~nm}$ per minute until a final thickness of $50 \mathrm{~nm}$. Moreover, the reproducibility of this SERS for chemical and biological samples have been experimentally validated previously [59]. This SERS design was found to have more than one million times Raman enhancement and works effectively to trap EVs in its hotspot areas [25]. Scanning electron microscopy (SEM) of $300 \mathrm{~nm}$ diameter polystyrene beads released on the SERS substrate was performed using a Hitachi SU-70 SEM to illustrate its trapping capability for EV-sized particles (Fig. 1 (a)) and optical microscopy demonstrates the consistent and tightly packed patterning of cup-shaped structures across the entire surface (Fig. 1 (b)). Atomic force microscopy (AFM) was also performed to further validate the nanocup structure of the fabricated SERS substrate using an Asylum Cypher ES AFM system.

For all SERS measurements, $1 \mu \mathrm{l}$ of EVs (in an ultrapure water suspension) per square millimeter, with the concentration of $\approx 1 \times 10^{10} \mathrm{EVs}$ per millilitre, were dropped on the SERS surface and dried quickly in a $40{ }^{\circ} \mathrm{C}$ oven to avoid coffee ring effect and increase the homogeneity of the EVs' distribution on the SERS surface. Raman measurements were carried out using a Horiba LabRAM HR Evolution confocal Raman microscope by using $785 \mathrm{~nm}$ laser and $50 \times$ microscope objective. 50 SERS spectra were taken for each EV sample from $800-1800 \mathrm{~cm}^{-1}$, with a minimum distance of one laser spot size between acquisition locations. The laser power at the surface of SERS was controlled using neutral density filter and set to 10 percent of the maximum power $(100 \mathrm{~mW})$. A $10 \mathrm{sec}$ acquisition time for the detector was chosen per measurement. Then, the baseline was established and noise was removed automatically using previously established asymmetric least squares smoothing [61].

\section{Machine Learning Analyses}

All machine learning and multivariate analyses were performed using the established Python library, Sklearn [62]. Some rationale behind the classification methods are explained within results and discussion section for added clarity.

\section{Results And Discussion}

\section{A. EV Characterisation}

NTA data demonstrated that all UPEC EVs exhibited similar sizes, with an average mean diameter of $115.4+/-6.5 \mathrm{~nm}$ and mode of $89.7+/-4.5 \mathrm{~nm}$ (Fig. 2 (a)). Interestingly, the NTA size distribution for Nissle-R-SEC EVs showed noticeably larger particles with a mean diameter of $135.8 \mathrm{~nm}$ and mode of $101.2 \mathrm{~nm}$, while K12-RF-SEC yielded smaller EVs mean diameter of $71.1 \mathrm{~nm}$ and a mode of $62.9 \mathrm{~nm}$. Negative staining TEM imaging demonstrated similar EV morphologies for all samples, and representative TEM images for EVs from each E. coli strain are shown in Fig. 2 (b). However, the slight differences in particle size measured by NTA were not apparent in the TEM images, potentially due to the random adsorption to the copper grids.

The post-processed SERS spectra for all E. coli EVs investigated in this work are shown in Fig. 2 (c). These spectra were normalised using euclidean normalization and thus any information related to the amplitude of SERS spectra were removed. The mean, maximum/minimum and standard deviation around the mean was calculated and presented with red, yellow and cyan colour, respectively. The correspondence of Raman bands to the known types of biomolecules are shown using vertical colour bands [63]. As most EV spectral peaks are the result of contributions from the chemical bonds of many different biochemical species in combination, only general indications about their contents are noted. For both EV size distributions and SERS spectra, mean, $\min / \max$, and standard deviation are represented as red, yellow, and cyan, respectively. 


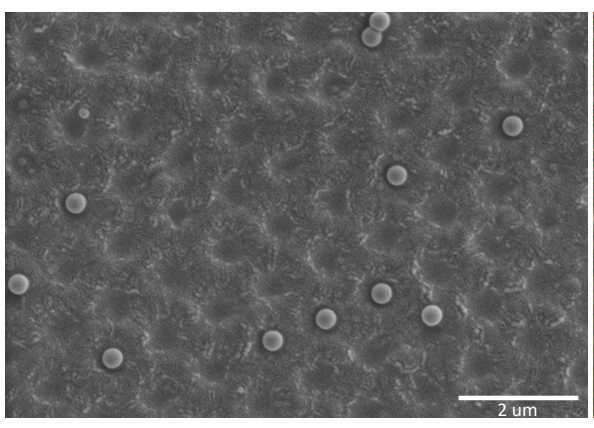

(a)

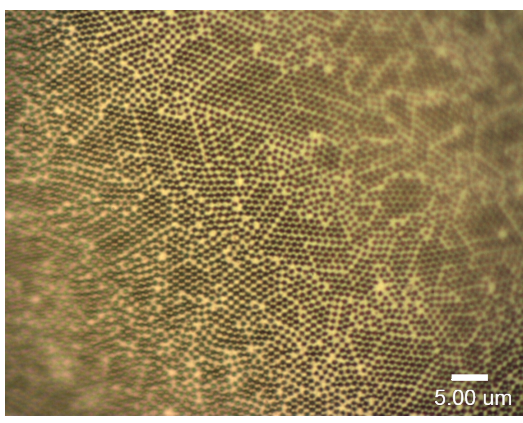

(b)

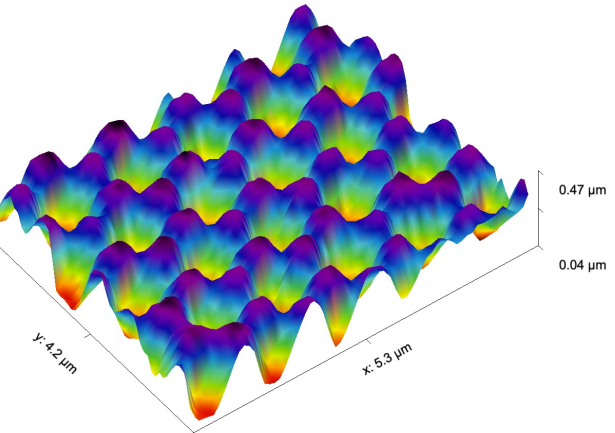

(c)

Fig. 1. Nanocup SERS substrate characterisation showing (a) SEM of $300 \mathrm{~nm}$ beads trapped in the nanocup SERS hot spots (scale bar $=4 \boldsymbol{\mu m}$ ), (b) optical microscopy image of tightly packed nanocup structures across the entire surface (scale bars $=\mathbf{5} \boldsymbol{\mu m}$ ), and (c) AFM of the nanocup SERS demonstrating the desired structure.

\section{B. Multivariate Analysis and Classification}

Samples were classified using standard machine learning algorithms in two different scenarios. In the first scenario, data from the three relevant pairwise comparisons based on strain, culture medium, or purification method were used for training and classification. This analysis was performed first to determine the effect of each individual parameter at a time. In the second scenario, the machine learning algorithms were trained to identify the EVs from all 6 subtypes simultaneously, such that the spectra of all EVs were used for training.

1) Paired Subset Classification: For the purpose of strain classification, paired subsets \{Nissle-R-SEC, UPEC-R-SEC and $\{\mathrm{K} 12-\mathrm{RF}-\mathrm{SEC}$, UPEC-RF-SEC $\}$ were used as they are different strains but were grown in identical culture medium and purified identically. Similarly, the paired subsets of \{UPEC-R-DG, UPEC-RF-DG $\}$ and \{UPEC-R-SEC, UPECRF-SEC $\}$ were used for the investigation of culture medium as they are the same strain and purified identically, but grown in different culture media. Lastly, \{UPEC-R-SEC, UPEC-RDG $\}$ and \{UPEC-RF-SEC, UPEC-RF-DG $\}$ were used for the evaluation of purification methods as they are the same strain and grown in identical culture medium, but purified using different methods.

To effectively present the variance of the obtained SERS spectra we investigated all the components of each spectra. Each of the obtained spectra consists of 1512 data points between Raman shifts of $800-1800 \mathrm{~cm}^{-1}$. These are, in fact, an array of 1512 dimensions and the vast number of dimensions severely limits the visualisation of the variance within the data. Principle Component Analysis (PCA) was used to transform the obtained spectra in a way which reduces the spectral dimensions while preserving the maximum variance between the data after transformation. This was done firstly for the sake of the visualisation and secondly as means of classification in lower dimensions between samples.

To demonstrate the possibility of the automatic classification, we used four different types of established machine learning algorithms, including Linear Discriminant Analysis (LDA), Gaussian Process Classifier (GPC), K-Nearest Neigh- bour (KNN), and Support Vector Classifier (SVC) with RBF kernel. All the machine learning algorithms were employed over the PCA transformed data to calculate the probability of each point in the PCA plane to be classified as the correct type within each subgroup. The average SERS spectra for K12-RFSEC and UPEC-RF-SEC, PCA transformation, and probability distribution obtained using machine learning algorithms, as well as first and second PCA scores are depicted in Fig. 3. Similar results of classification and PCA transformation for the other pairwise strain comparison, culture medium comparisons, and purification comparisons are available in Supplementary Fig. 1-5.

For the paired comparison of K12-RF-SEC and UPECRF-SEC (Fig. 3), clear differences in several peaks can be visually discerned, resulting in an expected and highly efficient classification by all the machine learning algorithms tested. By comparison, relatively subtle differences can be seen in the averaged SERS spectra for Nissle-R-SEC and UPEC-RSEC (Supplementary Fig 1), and while the classification is not as efficient, all the machine learning algorithms tested were still largely effective. For pairwise comparisons between plain and iron supplemented culture media (Supplementary Fig 23 ), clear differences in several peaks can be easily discerned, resulting in highly efficient classification in both cases. Lastly, differences between SEC and DG-purified UPEC EVs from both types of culture media can also be effectively classified (Supplementary Fig 4-5), although not quite to the level of efficiency as when comparing different culture media.

2) Classification of All Samples: Based on the initial success of classification using simple pairwise comparisons, a more challenging investigation of classifying all the samples simultaneously was explored. For the purpose of whole data classification, we first trained all the machine learning algorithms over each of the normalised spectra used to produce Fig. 2 (c) without PCA transformation. The confusion matrices produced when $60 \%$ of the data was used for training and the other $40 \%$ was used as a testing set are shown in Fig. 4 for the all chosen machine learning algorithms used previously, in addition Artificial Neural Network (ANN) and 


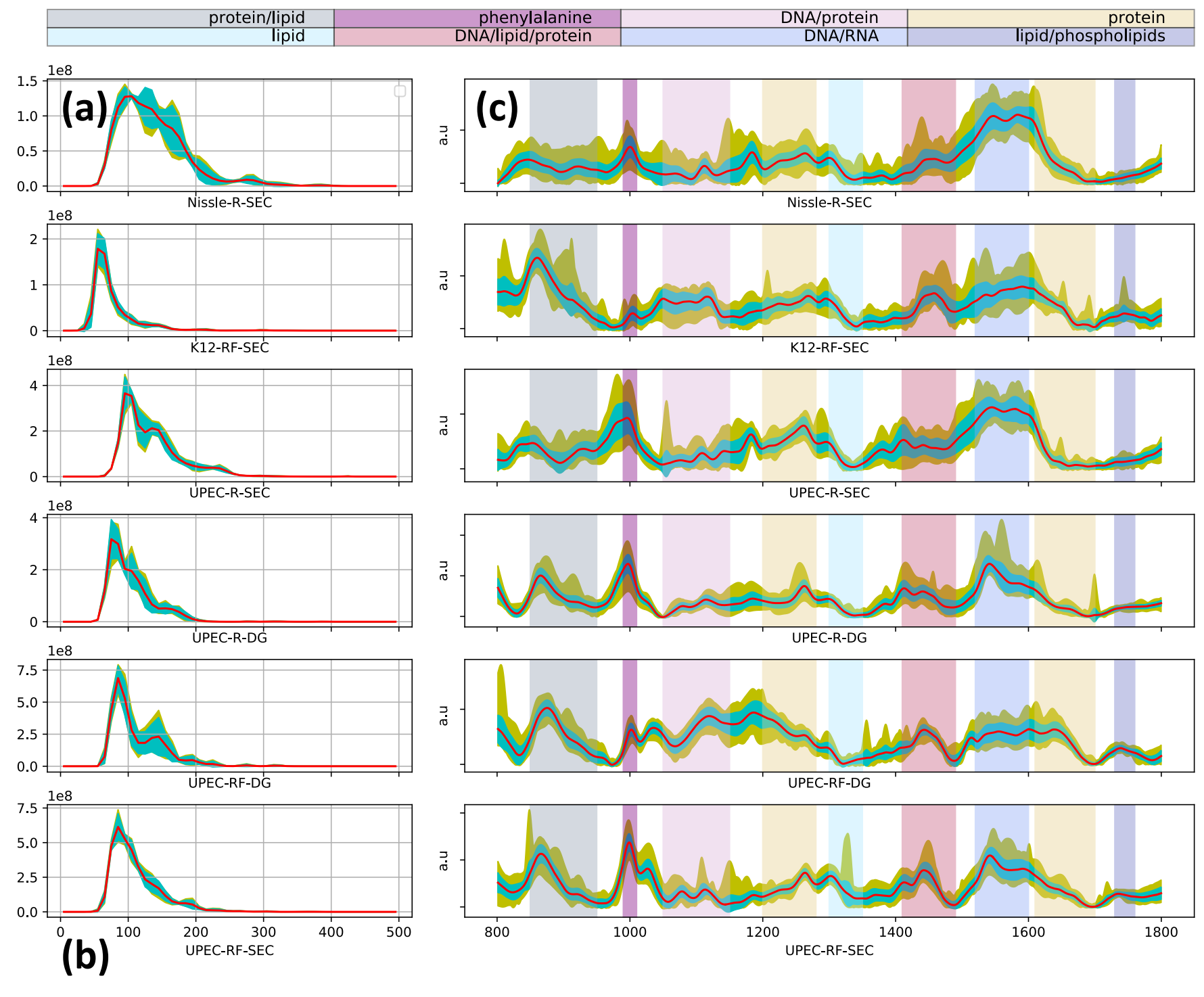

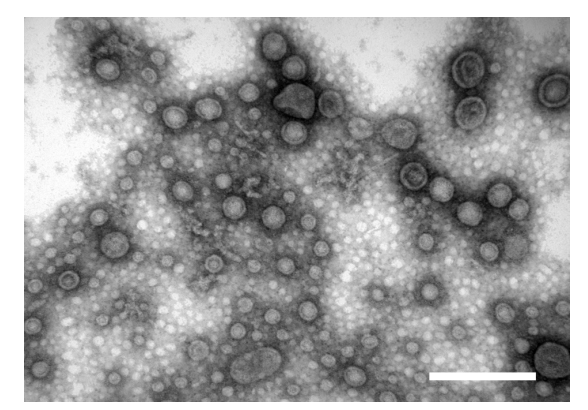

UPEC-R-DG

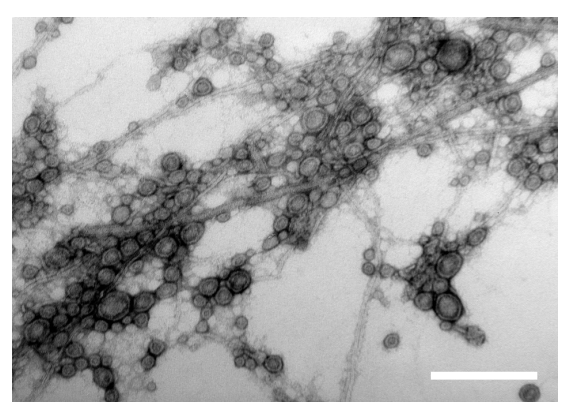

Nissle-R-SEC

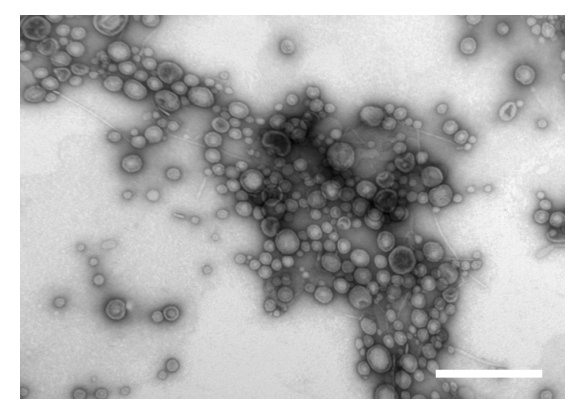

K12-RF-SEC

Fig. 2. EV characterisation showing (a) size distributions of all bacterial EV samples from NTA, (b) representative TEM of UPEC-R-DG, Nissle-RSEC, and K12-RF-SEC E. coli EVs (scale bars $=200 \mathrm{~nm}$ ), and (c) acquired SERS spectra of all E. coli EV samples after baseline establishment and denoising with mean, $\mathrm{min} / \mathrm{max}$, and standard deviation represented as red, yellow, and cyan, respectively.

Random Forest (RF). Of the six classification methods tested, five produced efficiencies above 90\%, with GPC, SVC, and ANN all resulting in greater than $95 \%$. LDA performed the worst, but still produced a classification efficiency of $86.8 \%$. Collectively, these results demonstrate the power of combining SERS with machine learning, as even subtle differences be- tween normalised bacterial EV fingerprints can enable highly efficient classification, even from the same species of bacteria.

We also performed the same algorithms on the PCAtransformed data. Cumulative explained variance and the first, second, and 100th PCA scores are depicted in Fig. 5 (a), (b), (c) and (d), respectively. The accuracy of SVC and ANN 

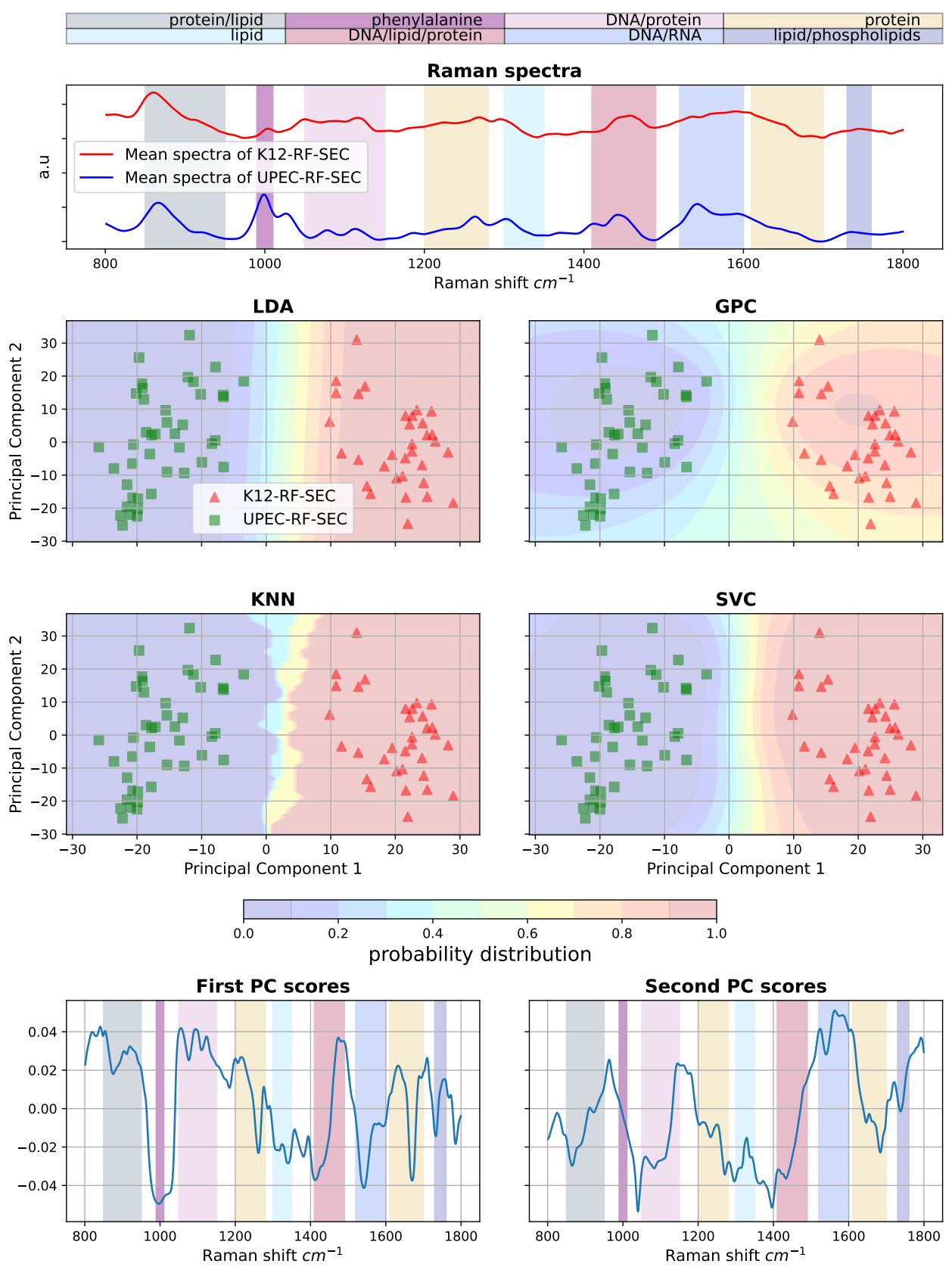

Fig. 3. Machine learning results for classification by strain between K12-RF-SEC and UPEC-RF-SEC showing (top) averaged EV SERS spectra, (middle) Linear Discriminant Analysis (LDA), Gaussian Process Classifier (GPC), K-nearest neighbour (KNN), and Support Vector Classifier (SVC), and (bottom) first and second PC scores.

machine learning algorithms when different PCA dimensions were considered for training are shown in Fig. 5 (e) and (f), demonstrating that the acceptable accuracy is achieved when few (5-10) PCs are used. Interestingly, increasing the number of components beyond a certain point does not lead to better classification result. One of the main reasons for this phenomena may be the natural variance in the E. coli $\mathrm{EV}$ biochemical contents within each subtype and the noise level due to weak Raman intensity of EVs. In fact, the $100^{\text {th }}$ PC depicted in Fig. 5 (d) contains little biochemical information about the EVs and thus adding more PCs for classification purposes can actually lead to weaker classification results.
3) Effects of Culture Medium on SERS Spectra: To further investigate the effect of culture medium on the SERS spectra, which is known to significantly affect the proteome of E. coli EVs according to a previous study [33], two dimensional PCA transformation of the whole data set is plotted in Fig. 6 (a) with the plain (R) and iron(III) chloride-supplemented (RF) medium marked by $\{$ red, green, blue $\}$ and \{purple, orange, brown $\}$, respectively. Clearly, the first principal component can effectively separate the data of $E$. coli EVs produced in the different media. This is likely due to the fact that the SERS spectra of EVs is mostly determined by the surface biochemical makeup of the EVs, which appears to be heavily influenced by the culture medium. In this study, the spectra 


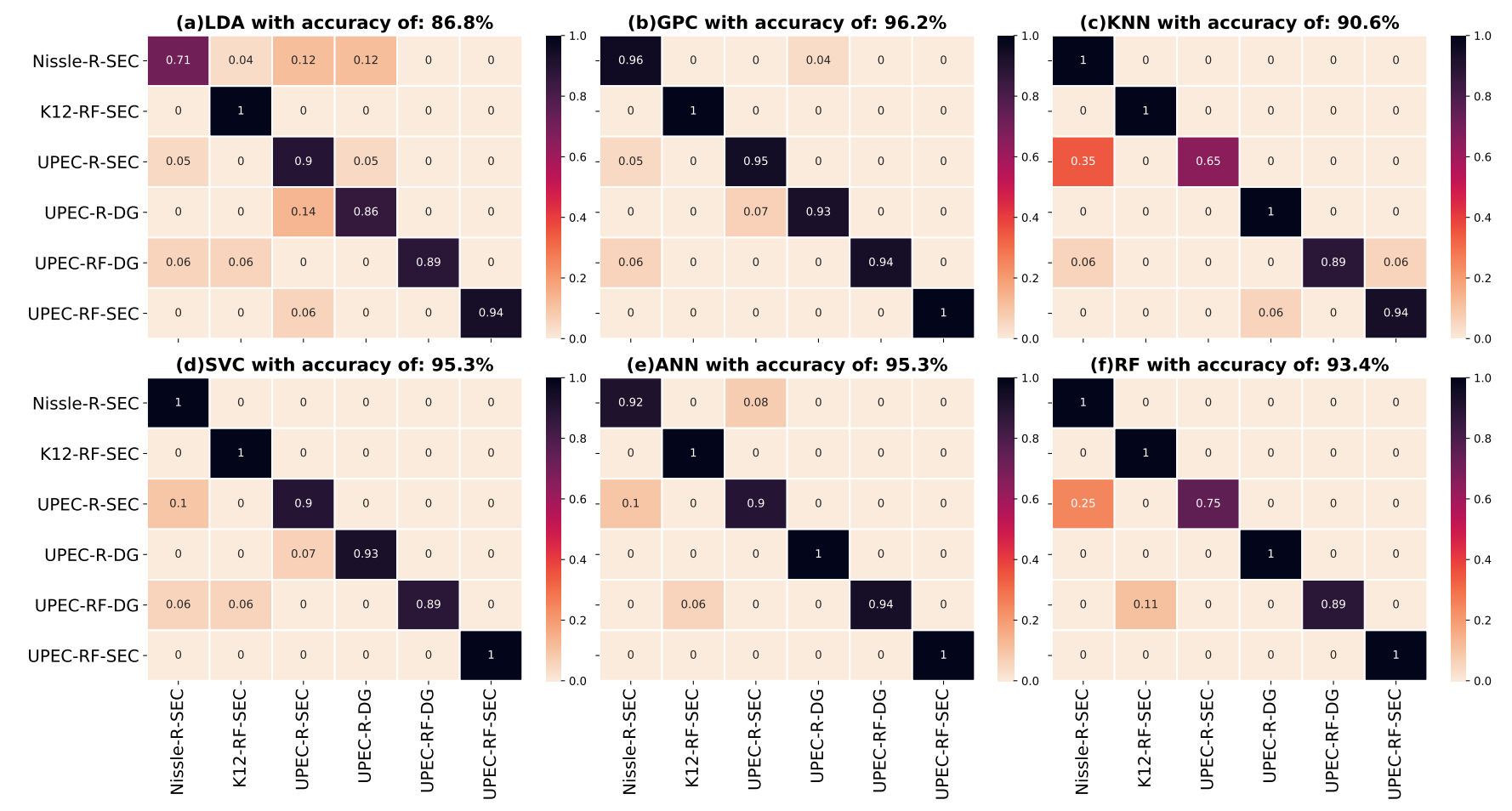

Fig. 4. Confusion matrices showing classification efficiency for all E. coli EVs using different machine learning methods, including (a) LDA, (b) GPC, (c) KNN, (d) SVC, (e) ANN and (f) RF.

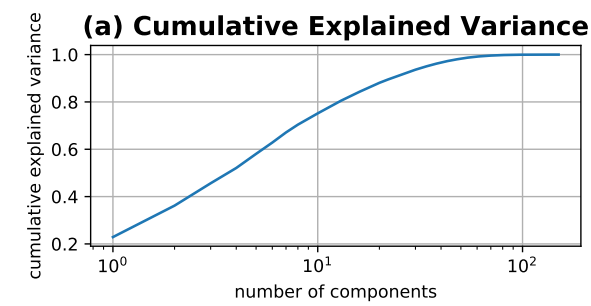

(c) Second Principal Component
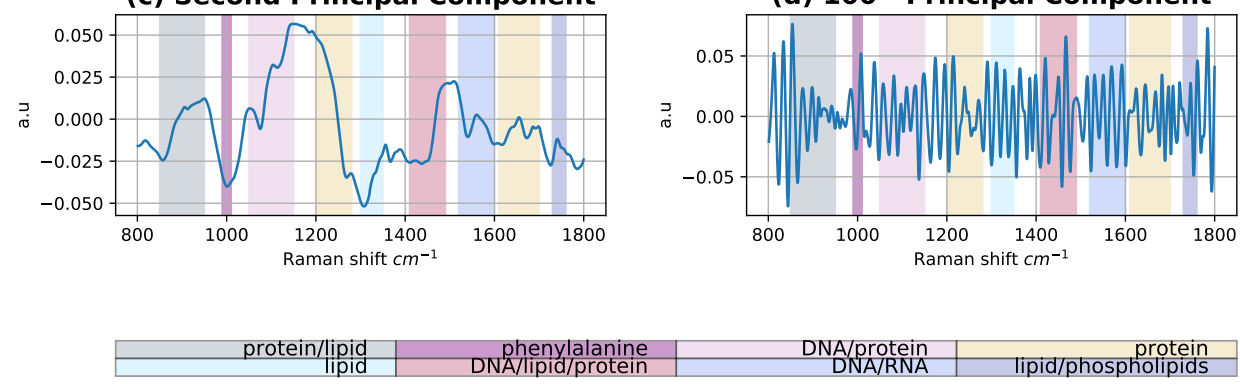

(e) SVC accuracy

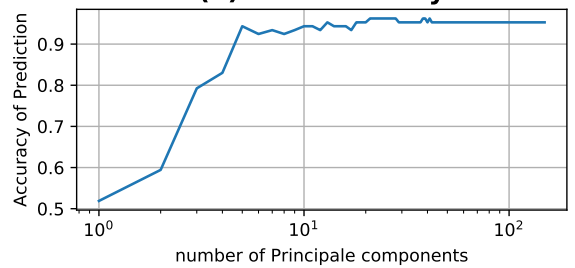

(b) First Principal Component

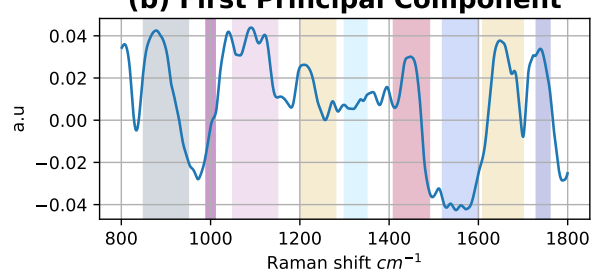

(d) $100^{\text {th }}$ Principal Component

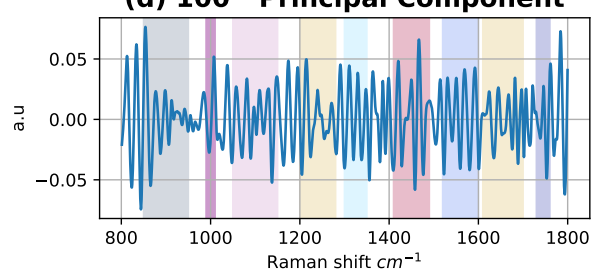

Fig. 5. All sample classification following PCA transformation showing (a) cumulative explained variance vs the number of principal components, scores of (b) first, (c) second, and (d) $\mathbf{1 0 0}^{\text {th }}$ principal components, (e) SVC and (f) ANN accuracy when different number of principal components are considered. 
(a) PCA

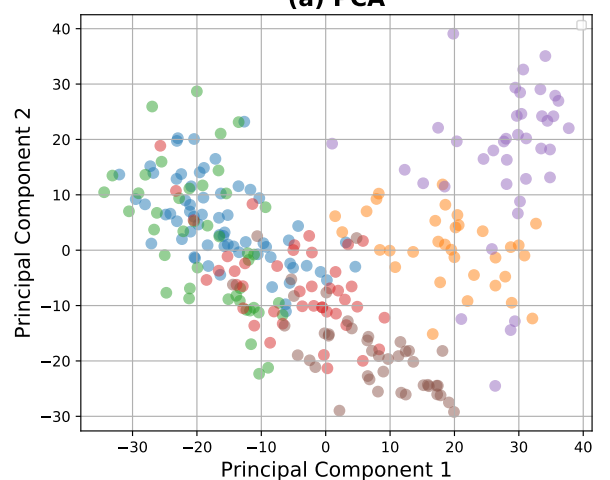

(b) t-SNE

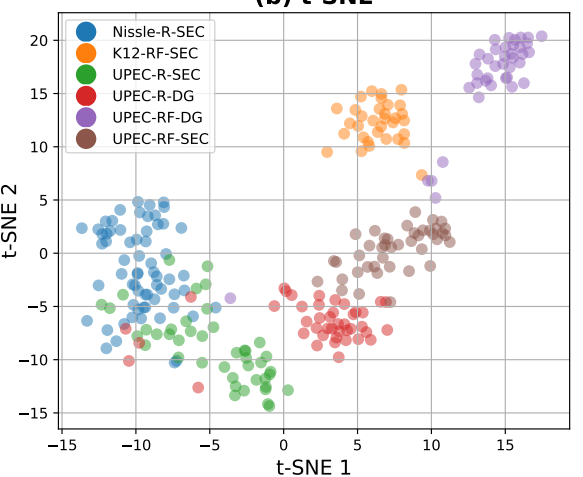

(c) UMAP

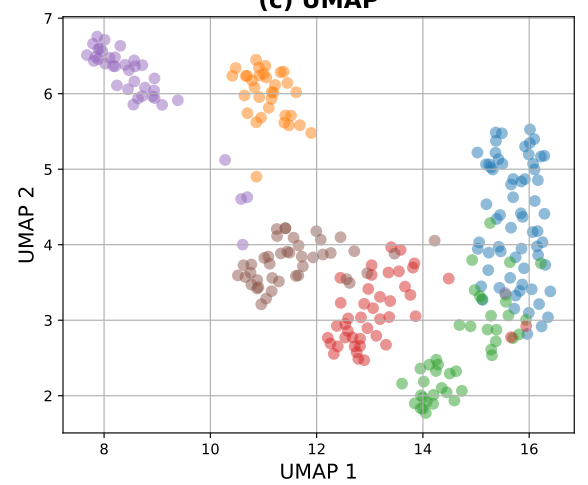

Fig. 6. Result of the manifold machine learning approaches comparing (a) conventional PCA to (b) t-SNE and (c) UMAP for all E. coli EV SERS spectra

below a Raman shift of $800 \mathrm{~cm}^{-1}$, which would contain peaks related to iron, were not acquired to focus on the EV biochemical contents rather than any free iron bound to the EV surfaces, while also increasing the speed of spectral acquisitions. This strong influence of culture media on the SERS spectra not only corroborates the previous study [33], but indicates that the type of fluid in which bacterial cells are cultured may need to be carefully considered in future EV SERS comparisons. This could be particularly important in clinical applications, as different bodily fluids may confound specific species or strain identification.

\section{Manifold Machine Learning for Improved Data Visualisation}

As shown in Fig. 6 (a), 2 dimensional PCA transformation can not effectively illustrate the variance of the data as the data points of each subgroup are mixed with no clear spacing between them. From the results of the classification algorithms using PCA-transformed data in Fig. 5 (e) and (f), we can see that the 5 dimensional PCA transformation, however, results in a very good classification accuracy. In other words, in 5 dimensional PCA space, the data corresponding to each subtype of $E$. coli EVs are effectively separated with clear boundaries between them. Given the challenges conceptualizing anything beyond 3 dimensions, a better dimensional reduction technique could ideally reduce the dimensional complexity. To achieve this, we employed t-SNE [64] and UMAP [65] as unsupervised manifold machine learning and non-linear dimensional reduction techniques, with the results presented in Fig. 6 (b) and (c), respectively. In clear contrast to the standard PCA transformation (Fig. 6 (a)), the variance and differences between the spectra of each $E$. coli EV subtype are much more clear in two dimensional UMAP and t-SNE planes. These results encourage the future use of manifold machine learning and non-linear dimensional reduction techniques for EV SERS studies.

\section{Conclusions}

In this study we demonstrate, for the first time, that EVs from bacteria can be effectively characterised using SERS and that the acquired spectra have the potential to be used for classification purposes. Several parameters appear to influence the SERS spectra of E. coli EVs including strain, purification method, and culture medium, which can be classified using standard machine learning approaches. In addition, manifold machine learning algorithms t-SNE and UMAP, which to the best of our knowledge have not yet been applied to EV SERS analysis, appear to be very effective for dimension reduction and visualisation of all the obtained SERS spectra. Collectively, these findings establish the incredible sensitivity and potential utility of SERS for bacterial EV analysis, as classification-enabling differences were seen in each sample subtype, despite the fact that all of the samples tested were from the same species of bacteria.

Future SERS experiments will include EVs from other species of gram-negative bacteria, as well as gram-positive bacteria and mycobacteria. As each have different cell and EV membrane compositions and proposed EV biogenesis mechanisms, the different EV envelope and contents will likely affect the SERS spectra, enabling rapid and label-free classification approaches. In addition, the isolation of bacteriaspecific EVs from more complex samples like host bodily fluids could be performed by targeting appropriate bacterial EV surface antigens. As the field of bacterial EVs continues to grow, we anticipate that these findings will enable and encourage other researchers to examine bacterial EVs using this highly sensitive technique for a nearly limitless range of laboratory or clinical applications.

\section{ACKNOWLEDGEMENTS}

The authors would like to thank the Photon Factory, the Hub for Extracellular Vesicle Investigations, and The DoddWalls Centre for Photonic and Quantum Technologies for their continued support. This work was funded by the DoddWalls Centre for Photonic and Quantum Technologies, the Goodfellow Fund for the Support of Urological Research, the University of Auckland's School of Medicine PerformanceBased Research Fund, the Maurice and Phyllis Paykel Trust Project Grant, Lottery Health Research Grant, Health Research Council of New Zealand Explorer Grant, and Ministry of Business Innovation and Enterprise Smart Ideas Grant. 


\section{REFERENCES}

[1] A. T. Tu and A. Tu, "Raman spectroscopy in biology: principles and applications," 1982.

[2] M. Jermyn, K. Mok, J. Mercier, J. Desroches, J. Pichette, K. SaintArnaud, L. Bernstein, M.-C. Guiot, K. Petrecca, and F. Leblond, "Intraoperative brain cancer detection with raman spectroscopy in humans," Science translational medicine, vol. 7, no. 274, pp. 274ra19-274ra19, 2015.

[3] H. Chen, X. Li, N. Broderick, Y. Liu, Y. Zhou, J. Han, and W. Xu, "Identification and characterization of bladder cancer by low-resolution fiber-optic raman spectroscopy," Journal of biophotonics, vol. 11, no. 9, p. e201800016, 2018.

[4] D. Klein, R. Breuch, S. von der Mark, C. Wickleder, and P. Kaul, "Detection of spoilage associated bacteria using raman-microspectroscopy combined with multivariate statistical analysis," Talanta, vol. 196, pp. 325-328, 2019.

[5] M. Saleem, S. Ali, M. B. Khan, A. Amin, M. Bilal, H. Nawaz, and M. Hassan, "Optical diagnosis of hepatitis b virus infection in blood plasma using raman spectroscopy and chemometric techniques," Journal of Raman Spectroscopy, vol. 51, no. 7, pp. 1067-1077, 2020.

[6] C.-S. Ho, N. Jean, C. A. Hogan, L. Blackmon, S. S. Jeffrey, M. Holodniy, N. Banaei, A. A. Saleh, S. Ermon, and J. Dionne, "Rapid identification of pathogenic bacteria using raman spectroscopy and deep learning," Nature communications, vol. 10, no. 1, pp. 1-8, 2019.

[7] D. Klein, R. Breuch, S. von der Mark, C. Wickleder, and P. Kaul, "Detection of spoilage associated bacteria using raman-microspectroscopy combined with multivariate statistical analysis," Talanta, vol. 196, pp. 325-328, 2019.

[8] J. van de Vossenberg, H. Tervahauta, K. Maquelin, C. H. BlokkerKoopmans, M. Uytewaal-Aarts, D. van der Kooij, A. P. van Wezel, and B. van der Gaag, "Identification of bacteria in drinking water with raman spectroscopy," Analytical Methods, vol. 5, no. 11, pp. 2679-2687, 2013.

[9] D. Kusić, B. Kampe, P. Rösch, and J. Popp, "Identification of water pathogens by raman microspectroscopy," Water research, vol. 48, pp. 179-189, 2014.

[10] K. Hamasha, Q. I. Mohaidat, R. A. Putnam, R. C. Woodman, S. Palchaudhuri, and S. J. Rehse, "Sensitive and specific discrimination of pathogenic and nonpathogenic escherichia coli using raman spectroscopy - a comparison of two multivariate analysis techniques," Biomedical optics express, vol. 4, no. 4, pp. 481-489, 2013.

[11] K. Bradley, J. Williams, L. Burnsed, M. Lytle, M. McDermott, R. Mody, A. Bhattarai, S. Mallonee, E. Piercefield, C. McDonald-Hamm et al. "Epidemiology of a large restaurant-associated outbreak of shiga toxinproducing escherichia coli o111: Nm," Epidemiology \& Infection, vol. 140, no. 9, pp. 1644-1654, 2012.

[12] C. N. Berger, S. V. Sodha, R. K. Shaw, P. M. Griffin, D. Pink, P. Hand, and G. Frankel, "Fresh fruit and vegetables as vehicles for the transmission of human pathogens," Environmental microbiology, vol. 12, no. 9, pp. 2385-2397, 2010.

[13] P. L. Stiles, J. A. Dieringer, N. C. Shah, and R. P. Van Duyne, "Surfaceenhanced raman spectroscopy," Annu. Rev. Anal. Chem., vol. 1, pp. 601626,2008

[14] C. Xie, J. Mace, M. Dinno, Y. Li, W. Tang, R. Newton, and P. Gemperline, "Identification of single bacterial cells in aqueous solution using confocal laser tweezers raman spectroscopy," Analytical chemistry, vol. 77, no. 14, pp. 4390-4397, 2005.

[15] F. J. García-Vidal and J. Pendry, "Collective theory for surface enhanced raman scattering," Physical Review Letters, vol. 77, no. 6, p. 1163, 1996.

[16] J. Pendry, A. Aubry, D. Smith, and S. Maier, "Transformation optics and subwavelength control of light," science, vol. 337, no. 6094, pp. 549-552, 2012.

[17] E. Temur, İ. H. Boyacı, U. Tamer, H. Unsal, and N. Aydogan, "A highly sensitive detection platform based on surface-enhanced raman scattering for escherichia coli enumeration," Analytical and bioanalytical chemistry, vol. 397, no. 4, pp. 1595-1604, 2010.

[18] D. Yang, H. Zhou, C. Haisch, R. Niessner, and Y. Ying, "Reproducible e. coli detection based on label-free sers and mapping," Talanta, vol. 146, pp. 457-463, 2016.

[19] S. Zong, L. Wang, C. Chen, J. Lu, D. Zhu, Y. Zhang, Z. Wang, and Y. Cui, "Facile detection of tumor-derived exosomes using magnetic nanobeads and sers nanoprobes," Analytical Methods, vol. 8, no. 25, pp. 5001-5008, 2016.

[20] C. Lee, R. Carney, K. Lam, and J. W. Chan, "Sers analysis of selectively captured exosomes using an integrin-specific peptide ligand," Journal of Raman Spectroscopy, vol. 48, no. 12, pp. 1771-1776, 2017.
[21] Y.-F. Tian, C.-F. Ning, F. He, B.-C. Yin, and B.-C. Ye, "Highly sensitive detection of exosomes by sers using gold nanostar@ raman reporter@ nanoshell structures modified with a bivalent cholesterol-labeled dna anchor," Analyst, vol. 143, no. 20, pp. 4915-4922, 2018.

[22] Z. Wang, S. Zong, Y. Wang, N. Li, L. Li, J. Lu, Z. Wang, B. Chen, and Y. Cui, "Screening and multiple detection of cancer exosomes using an sers-based method," Nanoscale, vol. 10, no. 19, pp. 9053-9062, 2018.

[23] E. A. Kwizera, R. O’Connor, V. Vinduska, M. Williams, E. R. Butch, S. E. Snyder, X. Chen, and X. Huang, "Molecular detection and analysis of exosomes using surface-enhanced raman scattering gold nanorods and a miniaturized device," Theranostics, vol. 8, no. 10, p. 2722, 2018.

[24] T.-D. Li, R. Zhang, H. Chen, Z.-P. Huang, X. Ye, H. Wang, A.-M. Deng, and J.-L. Kong, "An ultrasensitive polydopamine bi-functionalized sers immunoassay for exosome-based diagnosis and classification of pancreatic cancer," Chemical science, vol. 9, no. 24, pp. 5372-5382, 2018.

[25] C. Lee, R. P. Carney, S. Hazari, Z. J. Smith, A. Knudson, C. S. Robertson, K. S. Lam, and S. Wachsmann-Hogiu, "3d plasmonic nanobowl platform for the study of exosomes in solution," Nanoscale, vol. 7, no. 20, pp. 9290-9297, 2015.

[26] K. Sivashanmugan, W.-L. Huang, C.-H. Lin, J.-D. Liao, C.-C. Lin, W.-C. $\mathrm{Su}$, and T.-C. Wen, "Bimetallic nanoplasmonic gap-mode sers substrate for lung normal and cancer-derived exosomes detection," Journal of the Taiwan Institute of Chemical Engineers, vol. 80, pp. 149-155, 2017.

[27] L. Tirinato, F. Gentile, D. Di Mascolo, M. Coluccio, G. Das, C. Liberale, S. Pullano, G. Perozziello, M. Francardi, A. Accardo et al., "Sers analysis on exosomes using super-hydrophobic surfaces," Microelectronic Engineering, vol. 97, pp. 337-340, 2012.

[28] J. Park, M. Hwang, B. Choi, H. Jeong, J.-h. Jung, H. K. Kim, S. Hong, J.-h. Park, and Y. Choi, "Exosome classification by pattern analysis of surface-enhanced raman spectroscopy data for lung cancer diagnosis," Analytical chemistry, vol. 89, no. 12, pp. 6695-6701, 2017.

[29] H. Shin, H. Jeong, J. Park, S. Hong, and Y. Choi, "Correlation between cancerous exosomes and protein markers based on surface-enhanced raman spectroscopy (sers) and principal component analysis (pca)," ACS sensors, vol. 3, no. 12, pp. 2637-2643, 2018.

[30] Z. Yan, S. Dutta, Z. Liu, X. Yu, N. Mesgarzadeh, F. Ji, G. Bitan, and Y.-H. Xie, "A label-free platform for identification of exosomes from different sources," ACS sensors, vol. 4, no. 2, pp. 488-497, 2019.

[31] T. Nagakubo, N. Nomura, and M. Toyofuku, "Cracking open bacterial membrane vesicles," Frontiers in Microbiology, vol. 10, p. 3026, 2020.

[32] Y. Chen, L. Liu, H. Fu, C. Wei, and Q. Jin, "Comparative proteomic analysis of outer membrane vesicles from shigella flexneri under different culture conditions," Biochemical and biophysical research communications, vol. 453, no. 4, pp. 696-702, 2014.

[33] J. Hong, P. Dauros-Singorenko, A. Whitcombe, L. Payne, C. Blenkiron, A. Phillips, and S. Swift, "Analysis of the escherichia coli extracellular vesicle proteome identifies markers of purity and culture conditions," Journal of Extracellular Vesicles, vol. 8, no. 1, p. 1632099, 2019.

[34] C.-H. Chiu, Y.-T. Lee, Y.-C. Lin, S.-C. Kuo, Y.-S. Yang, Y.-C. Wang, Y.-H. Liu, J.-C. Lin, F.-Y. Chang, and T.-L. Chen, "Bacterial membrane vesicles from acinetobacter baumannii induced by ceftazidime are more virulent than those induced by imipenem," Virulence, vol. 11, no. 1, pp. 145-158, 2020.

[35] A. Malabirade, J. Habier, A. Heintz-Buschart, P. May, J. Godet, R. Halder, A. Etheridge, D. Galas, P. Wilmes, and J. V. Fritz, "The rna complement of outer membrane vesicles from salmonella enterica serovar typhimurium under distinct culture conditions," Frontiers in microbiology, vol. 9, p. 2015, 2018.

[36] C. Pérez-Cruz, F. Briansó, E. Sonnleitner, U. Bläsi, and E. Mercadé, "Rna release via membrane vesicles in pseudomonas aeruginosa pao1 is associated with the growth phase," Environmental Microbiology, 2021.

[37] B. S. R. D. Luz, A. Nicolas, S. Chabelskaya, V. d. R. Rodovalho, Y. Le Loir, V. A. d. C. Azevedo, B. Felden, and E. Guedon, "Environmental plasticity of the rna content of staphylococcus aureus extracellular vesicles," Frontiers in Microbiology, vol. 12, p. 482, 2021.

[38] T. Rojalin, B. Phong, H. J. Koster, and R. P. Carney, "Nanoplasmonic approaches for sensitive detection and molecular characterization of extracellular vesicles," Frontiers in Chemistry, vol. 7, p. 279, 2019.

[39] H. Shin, D. Seo, and Y. Choi, "Extracellular vesicle identification using label-free surface-enhanced raman spectroscopy: Detection and signal analysis strategies," Molecules, vol. 25, no. 21, 2020.

[40] J. R. White, P. Dauros-Singorenko, J. Hong, F. Vanholsbeeck, A. Phillips, and S. Swift, "The complex, bidirectional role of extracellular vesicles in infection," Biochemical Society Transactions, vol. 49, no. 2, pp. 881-891, 042021. 
[41] V. M. Chernov, A. A. Mouzykantov, N. B. Baranova, E. S. Medvedeva, T. Y. Grygorieva, M. V. Trushin, I. E. Vishnyakov, A. V. Sabantsev, S. N. Borchsenius, and O. A. Chernova, "Extracellular membrane vesicles secreted by mycoplasma acholeplasma laidlawii pg8 are enriched in virulence proteins," Journal of Proteomics, vol. 110, pp. 117-128, 2014.

[42] Y. Liu, K. A. Y. Defourny, E. J. Smid, and T. Abee, "Gram-positive bacterial extracellular vesicles and their impact on health and disease," Frontiers in Microbiology, vol. 9, p. 1502, 2018.

[43] P. Dauros-Singorenko, J. Hong, S. Swift, A. Phillips, and C. Blenkiron, "Effect of the extracellular vesicle rna cargo from uropathogenic escherichia coli on bladder cells," Frontiers in Molecular Biosciences, vol. 7, p. 269, 2020.

[44] P. Briaud and R. K. Carroll, "Extracellular vesicle biogenesis and functions in gram-positive bacteria," vol. 88, no. 12, 2020.

[45] C.-s. Kang, M. Ban, E.-J. Choi, H.-G. Moon, J.-S. Jeon, D.-K. Kim, S.K. Park, S. G. Jeon, T.-Y. Roh, S.-J. Myung, Y. S. Gho, J. G. Kim, and Y.-K. Kim, "Extracellular vesicles derived from gut microbiota, especially akkermansia muciniphila, protect the progression of dextran sulfate sodium-induced colitis," PLOS ONE, vol. 8, pp. 1-11, 2013.

[46] J. C. Caruana and S. A. Walper, "Bacterial membrane vesicles as mediators of microbe - microbe and microbe - host community interactions," Frontiers in Microbiology, vol. 11, p. 432, 2020.

[47] C. Coelho and A. Casadevall, "Answers to naysayers regarding microbial extracellular vesicles," Biochemical Society Transactions, vol. 47, no. 4, pp. 1005-1012, 2019.

[48] L. Brown, J. M. Wolf, R. Prados-Rosales, and A. Casadevall, "Through the wall: extracellular vesicles in gram-positive bacteria, mycobacteria and fungi," Nature Reviews Microbiology, vol. 13, no. 10, pp. 620-630, 2015.

[49] C. Schwechheimer and M. J. Kuehn, "Outer-membrane vesicles from gram-negative bacteria: biogenesis and functions," Nature reviews microbiology, vol. 13, no. 10, pp. 605-619, 2015.

[50] S. Erdmann, B. Tschitschko, L. Zhong, M. J. Raftery, and R. Cavicchioli, "A plasmid from an antarctic haloarchaeon uses specialized membrane vesicles to disseminate and infect plasmid-free cells," Nature microbiology, vol. 2, no. 10, pp. 1446-1455, 2017.

[51] C. Coelho, L. Brown, M. Maryam, R. Vij, D. F. Smith, M. C. Burnet, J. E. Kyle, H. M. Heyman, J. Ramirez, R. Prados-Rosales et al., "Listeria monocytogenes virulence factors, including listeriolysin o, are secreted in biologically active extracellular vesicles," Journal of Biological Chemistry, vol. 294, no. 4, pp. 1202-1217, 2019.

[52] J. Lee, O. Y. Kim, and Y. S. Gho, "Proteomic profiling of gramnegative bacterial outer membrane vesicles: current perspectives," PROTEOMICS-Clinical Applications, vol. 10, no. 9-10, pp. 897-909, 2016.

[53] M. Potter, C. Hanson, A. J. Anderson, E. Vargis, and D. W. Britt, "Abiotic stressors impact outer membrane vesicle composition in a beneficial rhizobacterium: Raman spectroscopy characterization," Scientific Reports, vol. 10, no. 1, p. 21289, 2020.

[54] S. Knapp, J. Hacker, T. Jarchau, and W. Goebel, "Large, unstable inserts in the chromosome affect virulence properties of uropathogenic escherichia coli o6 strain 536." Journal of bacteriology, vol. 168, no. 1, pp. 22-30, 1986.

[55] A. Nissle, "Die antagonistische behandlung chronischer darmstörungen mit colibakterien," Med Klin, vol. 2, pp. 29-33, 1918.

[56] G. Sezonov, D. Joseleau-Petit, and R. D’Ari, "Escherichia coli physiology in luria-bertani broth," Journal of Bacteriology, vol. 189, no. 23, pp. 8746-8749, 2007. [Online]. Available: https://jb.asm.org/content/189/23/8746

[57] P. Mao, C. Liu, G. Favraud, Q. Chen, M. Han, A. Fratalocchi, and S. Zhang, "Broadband single molecule sers detection designed by warped optical spaces," Nature communications, vol. 9, no. 1, pp. 18, 2018 .

[58] M. Kazemzadeh, N. Broderick, W. Xu, and K. Z. Shoshtari, "Design and optimization of broadband and transparent sers based on transformation optics," in SPIE Micro+ Nano Materials, Devices, and Applications 2019, vol. 11201. International Society for Optics and Photonics, 2019, p. 112011L.

[59] M. Kazemzadeh, C. L. Hisey, A. Artuyants, C. Blenkiron, L. W. Chamley, K. Zargar-Shoshtari, W. Xu, and N. G. Broderick, "Space curvature-inspired nanoplasmonic sensor for breast cancer extracellular vesicle fingerprinting and machine learning classification," Biomedical Optics Express, vol. 12, no. 7, pp. 3965-3981, 2021.

[60] M. Kahraman, P. Daggumati, O. Kurtulus, E. Seker, and S. WachsmannHogiu, "Fabrication and characterization of flexible and tunable plasmonic nanostructures," Scientific reports, vol. 3, no. 1, pp. 1-9, 2013.
[61] P. H. Eilers and H. F. Boelens, "Baseline correction with asymmetric least squares smoothing," Leiden University Medical Centre Report, vol. 1, no. 1, p. 5, 2005.

[62] F. Pedregosa, G. Varoquaux, A. Gramfort, V. Michel, B. Thirion, O. Grisel, M. Blondel, P. Prettenhofer, R. Weiss, V. Dubourg, J. Vanderplas, A. Passos, D. Cournapeau, M. Brucher, M. Perrot, and E. Duchesnay, "Scikit-learn: Machine learning in Python," Journal of Machine Learning Research, vol. 12, pp. 2825-2830, 2011.

[63] Z. Movasaghi, S. Rehman, and I. U. Rehman, "Raman spectroscopy of biological tissues," Applied Spectroscopy Reviews, vol. 42, no. 5, pp. 493-541, 2007.

[64] L. Van der Maaten and G. Hinton, "Visualizing data using t-sne." Journal of machine learning research, vol. 9, no. 11, 2008.

[65] L. McInnes, J. Healy, and J. Melville, "Umap: Uniform manifold approximation and projection for dimension reduction," arXiv preprint arXiv:1802.03426, 2018. 